\title{
Preoperative Prognostic Nutritional Index Predicts Long-Term Surgical Outcomes in Patients with Esophageal Squamous Cell Carcinoma
}

\author{
Noriyuki Hirahara $^{1}$ - Yoshitsugu Tajima ${ }^{1}$ Yusuke Fujii ${ }^{1}$ Shunsuke Kaji ${ }^{1}$. \\ Tetsu Yamamoto $^{1} \cdot$ Ryoji Hyakudomi $^{1}$ ' Takahito Taniura $^{1} \cdot$ Yoshiko Miyazaki $^{1}$. \\ Takashi Kishi $^{1} \cdot$ Yasunari Kawabata $^{1}$
}

Published online: 31 December 2017

(C) The Author(s) 2017. This article is an open access publication

\begin{abstract}
Background The purpose of the present study is to investigate the utility of prognostic nutritional index (PNI) as a simple and readily available marker in esophageal squamous cell carcinoma (ESCC).

Methods We retrospectively analyzed 169 patients who underwent potentially curative esophagectomy, for histologically verified ESCC. We decided to set the optimal cutoff value for preoperative PNI levels at 49.2, based on the cancer-specific survival (CSS) and the overall survival (OS) by receiver operating characteristic curve analysis.

Results Multivariate logistic regression analysis identified that TNM pStage III [hazard ratio (HR) 3.261, $p<0.0001$ ] and PNI $<49.2$ (HR 3.887, $p<0.0001$ ) were confirmed as independent poor predictive factors for CSS, and age $>70$ (HR 2.024, $p<0.0042$ ), TNM pStage III (HR 2.510, $p=0.0002$ ), and PNI $<49.2$ (HR 2.248, $p=0.0013$ ) were confirmed as independent poor predictive factors for OS. In non-elderly patients, TNM pStage III (CSS; HR 3.488, $p<0.0001$, OS; HR 2.615, $p=0.0007$ ) and PNI $<49.2$ (CSS; HR 3.849, $p<0.0001$, OS; HR $2.275, p=0.001$ ) were confirmed as independent poor predictive factors for CSS, and OS when multivariate logistic regression analysis was applied. But in elderly patients, univariate analyses demonstrated that the TNM pStage III was the only significant risk factor for CSS (HR 3.701, $p=0.0057$ ) and OS (HR 1.974, $p=0.0224$ ).

Conclusions The PNI was a significant and independent predictor of CSS and OS of ESCC patients after curative esophagectomy. The PNI was cost-effective and readily available, and it could act as a marker of survival.
\end{abstract}

\section{Introduction}

Esophageal cancer is a disease of the elderly, with peak incidence occurring in patients in their $70 \mathrm{~s}$, and the elderly population is rapidly increasing in worldwide. Early detection and treatment confers the greatest chance of longterm survival in patients with esophageal cancer. However, a common problem among esophageal cancer patients (especially the elderly) is low tolerance to the available

Noriyuki Hirahara

norinorihirahara@yahoo.co.jp

1 Department of Digestive and General Surgery, Faculty of Medicine, Shimane University, 89-1 Enya-cho, Izumo, Shimane 693-8501, Japan treatments, despite improvements in surgical techniques and perioperative care with reduced perioperative mortality after esophagectomy. Therefore, there is a continuing interest in prognostic factors to identify patients who are at greater risks of recurrence in order to better tailor individual treatments to those who are more likely to benefit from them. Progression and prognosis of cancer has been shown to be impacted by both the tumor features as well as the patient's nutritional and immunologic status $[1,2]$. Several screening tools are now available for assessing the preoperative nutritional status of patients with cancer, including the subjective global assessment, nutritional risk scoring 2002, body mass index, Glasgow Prognostic Score, and prognostic nutritional index (PNI) [3-6]. Many studies have addressed the association between preoperative 
nutritional status and postoperative outcomes. However, there is little data to show the impact of nutritional status on long-term outcomes in patients undergoing radical thoracoscopic esophagectomy for esophageal squamous cell carcinoma (ESCC) [7, 8].

The PNI was originally developed to predict the risk of postoperative morbidity and mortality after gastrointestinal surgery [9]. However, the method for calculating PNI is complicated and difficult to use routinely in clinical practice. In contrast, the simplified PNI proposed by Onodera et al. [6] can be easily measured because it is based on only two laboratory parameters, namely the serum albumin level and the peripheral blood lymphocyte count. Albumin is a widely used parameter of nutrition, and its levels have been shown to correlate well with postoperative complications and long-term outcomes in several malignancies [10]. Similarly, reduced lymphocyte count and function also correlate with poor prognosis, by enabling cancer cells to escape immune surveillance [11]. PNI, therefore, is a favorable indicator and is more reflective of the overall status of cancer patients.

In the management of cancer patients, there has been a continuing interest in preoperative prognostic indicators that can allow more accurate patient stratification, precise clinical decision-making, and improvement of short- and long-term outcomes. The TNM classification is widely used as a good standard prognostic indicator of patients with cancer [12]. However, since it includes pathological findings of the tumor, a conclusive classification can only be made postoperatively. In contrast, the PNI is based on the preoperative laboratory data alone and could be a comprehensive indicator of postoperative morbidity, mortality, and prognosis of cancer patients prior to surgery.

Therefore, we have demonstrated the prognostic significance of the simplified PNI in overall and age-stratified ESCC patients with a low versus high PNI, and evaluated its potential as a useful surrogate marker for predicting postoperative patient survival.

\section{Methods}

\section{Patients}

We retrospectively reviewed a database containing data from 169 patients who underwent potentially curative esophagectomy with R0 resection, for histologically verified ESCC in our institute, between January 2006 and December 2015. R0 resection was defined as complete tumor removal without the involvement of any microscopic resection margin. Video-assisted or thoracoscopic subtotal esophagectomy with three-field lymph node dissection was performed for all patients, followed by laparoscopic gastric surgery with an elevation of the gastric conduit to the neck via the posterior mediastinal approach or retrosternal approach with an end-to-end anastomosis of the cervical esophagus and gastric conduit. The patients' clinical characteristics, laboratory data, treatment regimen, and pathological data were obtained from their medical records. None of the patients had clinical signs of infection or other systemic inflammatory conditions preoperatively. In this study, we excluded patients who had received preor postoperative adjuvant chemotherapy and/or radiotherapy.

We introduced a perioperative multidisciplinary management team, including surgeon, anesthesiologist, dental hygienist doctor, pharmacist, nutritionist, and rehabilitation technician, and certified expert surgical nurse, which aimed to decrease the incidence rate of postoperative complications. This team mainly managed dental cleaning, medication assistance, physical exercise and rehabilitation, respiratory training, and nutritional support. We provided preoperative enteral nutrition to optimize preoperative condition as possible.

The severity of postoperative complications was evaluated according to the Clavien-Dindo classification, and grade II or higher was recorded as a postoperative complication [13].

We evaluated cancer-specific survival (CSS) and overall survival (OS) as the endpoints of the study. The observation period started from the day of the operation and lasted for up to 5 years, loss to follow-up, withdrawal of consent, or until death. CSS was defined as the interval from the date of operation to the date of cancer-specific death or the last follow-up. Two patients who died of myocardial infarction within 60 days after esophagectomy were excluded from the analysis. We defined 'elderly' patients as those aged 70 years or older and 'non-elderly' as those aged less than 70 years of age [14].

Permission to perform this retrospective study was obtained from the ethical board of our institution and the study was conducted in accordance with the Declaration of Helsinki.

\section{Preoperative calculation of the PNI and its cutoff value}

The preoperative PNI was calculated using the following formula: $10 \times$ serum albumin $(\mathrm{g} / \mathrm{dl})+0.005 \times$ total lymphocyte count (per $\mathrm{mm}^{3}$ ) in peripheral blood [6].

The receiver operating characteristics (ROC) curve of preoperative PNI levels was generated for multiple logistic regression analysis using CSS and OS. The area under the curve (AUC) estimation was used to assess the predictive ability of the PNI. 
We decided to set the optimal cutoff value for preoperative PNI levels at 49.2 in this study, based on the CSS (sensitivity: $52.99 \%$; specificity: $80.77 \%$; AUC of the ROC curve: 0.653 ) and the OS (sensitivity: $52.63 \%$; specificity: $70.27 \%$; AUC of the ROC curve: 0.6132 ) at 5 years after surgery (Fig. 1). Based on their PNI values, patients were categorized as having a high PNI (49.2 or greater) or as having a low PNI (less than 49.2).

\section{TNM pathological Stage (pStage)}

The pathological classification of the primary tumor, the degree of lymph node involvement, and the presence of organ metastasis were determined according to the TNM classification system (7th edition of the cancer staging manual of the American Joint Committee on Cancer) [15].

\section{Statistical analysis}

The mean and standard deviation were calculated, and the differences were analyzed using Student's $t$ test. Differences between the various clinicopathological features were analyzed using the Chi-square test. The CSS was analyzed using Kaplan-Meier statistics, and inter-group differences were assessed using the log-rank test.

Univariate analyses were performed to determine variables associated with CSS. Variables with $p$ values $<0.05$ in the univariate analyses were included in a multivariate logistic regression analysis. The potential prognostic factors assessed were as follows: age ( $<70$ vs. $\geq 70$ years), sex (female vs. male), TNM pStage (I/II vs. III), tumor size $(<3$ vs. $\geq 3 \mathrm{~cm}$ ), operation time ( $<600$ vs. $\geq 600 \mathrm{~min})$, intraoperative blood loss ( $<500 \mathrm{vs.} \geq 500 \mathrm{~mL}$ ), serum squamous cell carcinoma antigen (SCC) value ( $<1.5$ vs. $\geq 1.5)$, and PNI ( $<49.2$ vs. $\geq 49.2)$.
All statistical analyses were performed using the statistical software JMP (version 11 for Windows; SAS Institute, Cary, $\mathrm{NC}$ ), and $p$ values $<0.05$ were considered statistically significant.

\section{Results}

\section{Relationship between PNI and clinicopathological features}

The correlation between the PNI and clinicopathological parameters in the 169 patients enrolled in this study is summarized in Table 1. The preoperative mean value of the PNI in this study was $47.3 \pm 6.2$, ranging from 26.8 to 65.7. Based on the cutoff value of $49.2,98$ patients (58\%) were in the low PNI category and 71 patients $(42 \%)$ were in the high PNI category. The results of our analysis showed that the PNI value correlated significantly with lymphocyte count $(p<0.0001)$, tumor size $(p=0.0327)$, depth of tumor $(p<0.0001)$, TNM pStage $(p=0.0005)$, SCC antigen level $(p=0.0072)$, albumin level $(p<0.0001)$, and CRP level $(p=0.0012)$.

\section{Prognostic factors for postoperative survival in patients with ESCC}

Univariate analyses identified advanced TNM pStage [hazard ratio (HR), 4.170; 95\% confidence interval (CI), 2.377-7.525; $p<0.0001$ ], large tumor size (HR 1.854; 95\% CI 1.093-3.678; $p=0.0233$ ), prolonged operation time (HR 1.756; 95\% CI 1.012-3.035; $p=0.0452$ ), and low PNI (HR 4.566; 95\% CI 2.375-9.674; $p<0.0001$ ) as significant risk factors for shorter CSS. The TNM pStage (HR 3.261; 95\% CI 1.808-6.043; $p<0.0001$ ) and PNI (HR 3.887; 95\% CI 1.999-8.309; $p<0.0001$ ) were
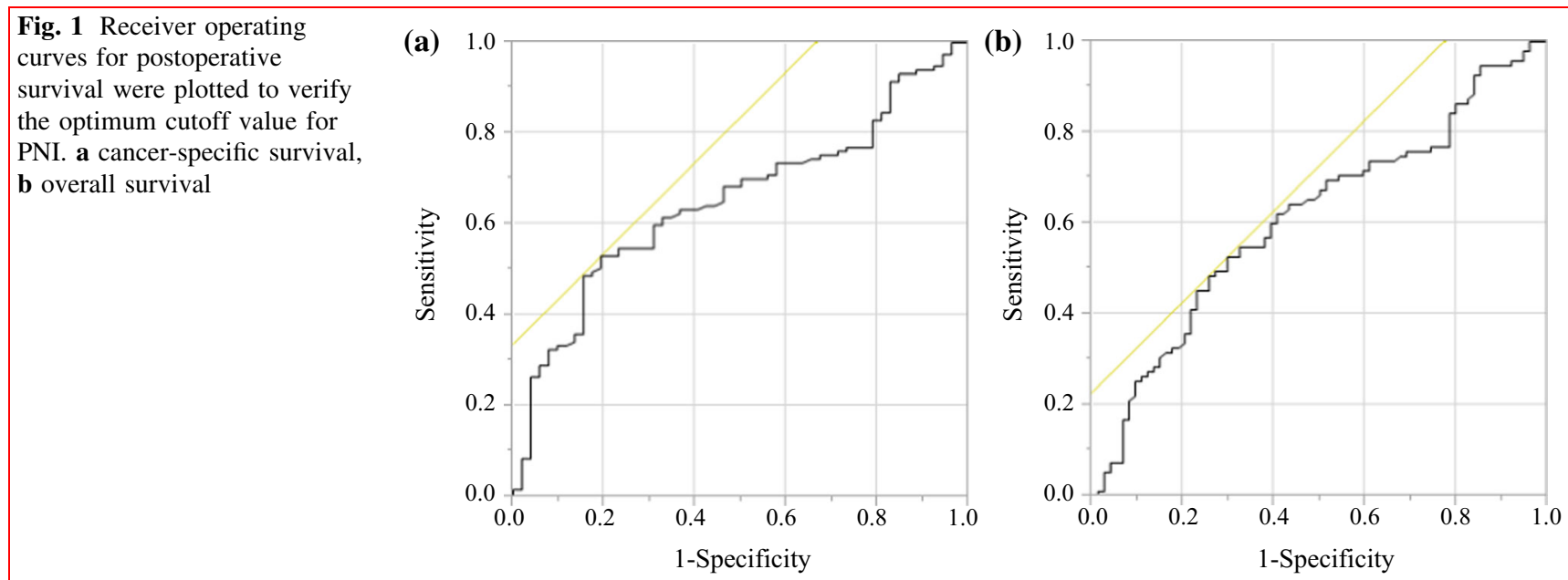
Table 1 Relationships between PNI and clinicopathological features in 169 patients with esophageal cancer

\begin{tabular}{|c|c|c|c|c|}
\hline \multirow[t]{2}{*}{ Characteristics } & \multirow[t]{2}{*}{ Total patients } & \multicolumn{3}{|l|}{ PNI } \\
\hline & & $\begin{array}{l}<49.2 \\
(n=98)\end{array}$ & $\begin{array}{l}\geq 49.2 \\
(n=71)\end{array}$ & $p$ value \\
\hline Age (years) & & $67.1 \pm 8.2$ & $65.4 \pm 8.0$ & 0.1804 \\
\hline Sex & & & & 0.628 \\
\hline Male & 150 & 86 & 64 & \\
\hline Female & 19 & 12 & 7 & \\
\hline WBC & & $5830.0 \pm 2404.6$ & $6233.9 \pm 1616.6$ & 0.2209 \\
\hline Neutrophil & & $3773.6 \pm 2197.3$ & $3669.2 \pm 1264.6$ & 0.7199 \\
\hline Lymphocyte & & $1396.4 \pm 562.7$ & $1931.8 \pm 599.2$ & $<0.0001$ \\
\hline Platelet & & $232.8 \pm 75.5$ & $225.5 \pm 50.2$ & 0.4796 \\
\hline Location of tumor & & & & 0.0696 \\
\hline $\mathrm{Ce}$ & 13 & 12 & 1 & \\
\hline $\mathrm{Ut}$ & 10 & 6 & 4 & \\
\hline Mt & 73 & 39 & 34 & \\
\hline $\mathrm{Lt}$ & 59 & 31 & 28 & \\
\hline $\mathrm{Ae}$ & 14 & 10 & 4 & \\
\hline Tumor size (mm) & & $5.27 \pm 4.98$ & $3.88 \pm 2.53$ & 0.0327 \\
\hline Depth of tumor & & & & $<0.0001$ \\
\hline T1a-1b & 72 & 27 & 45 & \\
\hline 2 & 13 & 8 & 5 & \\
\hline 3 & 67 & 48 & 19 & \\
\hline $4 a-4 b$ & 17 & 15 & 2 & \\
\hline Lymph node metastasis & & & & 0.1573 \\
\hline NO & 89 & 49 & 40 & \\
\hline$N 1$ & 48 & 28 & 20 & \\
\hline$N 2$ & 18 & 9 & 9 & \\
\hline$N 3$ & 14 & 12 & 2 & \\
\hline Pathological stage & & & & 0.0005 \\
\hline $1 a-1 b$ & 62 & 24 & 38 & \\
\hline $2 a-2 b$ & 39 & 28 & 11 & \\
\hline $3 a-3 c$ & 68 & 46 & 22 & \\
\hline Operation time $(\min )$ & & $656.1 \pm 196.1$ & $646.7 \pm 158.6$ & 0.7391 \\
\hline Intraoperative blood loss $(\mathrm{ml})$ & & $665.5 \pm 601.2$ & $580.0 \pm 612.3$ & 0.3666 \\
\hline SCC antigen & & $1.67 \pm 2.04$ & $0.97 \pm 0.85$ & 0.0072 \\
\hline Albumin & & $3.64 \pm 0.43$ & $4.32 \pm 0.30$ & $<0.0001$ \\
\hline CRP & & $0.658 \pm 1.098$ & $0.217 \pm 0.314$ & 0.0012 \\
\hline
\end{tabular}

confirmed as independent prognostic factors for CSS when multivariate logistic regression analysis was applied (Table 2).

Univariate analyses identified advanced age (HR 1.930; 95\% CI 1.205-3.061; $p=0.0066)$, advanced TNM pStage (HR 2.725; 95\% CI 1.714-4.349; $p<0.0001$ ), large tumor size (HR 1.868; 95\% CI 1.149-3.141; $p=0.0112$ ), and low PNI (HR 2.612; 95\% CI 1.600-4.405; $p<0.0001$ ) as significant risk factors for shorter OS. The age (HR 2.024; 95\% CI $1.255-3.236 ; p=0.0042)$, TNM pStage (HR $2.510 ; 95 \%$ CI 1.555-4.064; $p=0.0002)$, and PNI (HR
2.248; 95\% CI 1.362-3.832; $p=0.0013$ ) were confirmed as independent prognostic factors for OS in multivariate logistic regression analysis (Table 3 ).

\section{Prognostic factors for postoperative survival in non- elderly patients with ESCC}

Among 110 non-elderly patients, univariate analyses identified advanced TNM pStage (HR 4.646; 95\% CI 2.281-10.027; $p<0.0001$ ), large tumor size (HR 2.872; 95\% CI 1.349-6.825; $p=0.0054$ ), and low PNI (HR 
Table 2 Prognostic factors for cancer-specific survival in patients with esophageal cancer

\begin{tabular}{|c|c|c|c|c|c|c|c|c|}
\hline \multirow[t]{2}{*}{ Variables } & \multirow[t]{2}{*}{ Patients $(n=169)$} & \multirow[t]{2}{*}{ Category or characteristics } & \multicolumn{3}{|c|}{ Univariate } & \multicolumn{3}{|c|}{ Multivariate } \\
\hline & & & HR & $95 \% \mathrm{CI}$ & $p$ value & HR & $95 \% \mathrm{CI}$ & $p$ value \\
\hline Gender & $19 / 150$ & (Female/male) & 1.14 & $0.499-3.290$ & 0.7765 & & & \\
\hline Age & $59 / 110$ & $(<70 / \geq 70)$ & 1.42 & $0.790-2.480$ & 0.2344 & & & \\
\hline TNM pStage & $101 / 68$ & $(1,2 / 3)$ & 4.17 & $2.377-7.525$ & $<0.0001$ & 3.261 & $1.808-6.043$ & $<0.0001$ \\
\hline Tumor size & $64 / 105$ & $(<3 / \geq 3)$ & 1.854 & $1.093-3.678$ & 0.0233 & 1.076 & $0.583-2.079$ & 0.8203 \\
\hline Operation time & $111 / 58$ & $(<600 / \geq 600)$ & 1.756 & $1.012-3.035$ & 0.0452 & 1.595 & $0.910-2.786$ & 0.1024 \\
\hline Intraoperative blood loss & $87 / 82$ & $(<500 / \geq 500)$ & 0.973 & $0.562-1.685$ & 0.9226 & & & \\
\hline PNI & $98 / 71$ & $(\geq 49.2 /<49.2)$ & 4.566 & $2.375-9.674$ & $<0.0001$ & 3.887 & $1.999-8.309$ & $<0.0001$ \\
\hline SCC & $126 / 43$ & $(<1.5 / \geq 1.5)$ & 1.169 & $0.584-2.171$ & 0.6425 & & & \\
\hline
\end{tabular}

Table 3 Prognostic factors for overall survival in patients with esophageal cancer

\begin{tabular}{|c|c|c|c|c|c|c|c|c|}
\hline \multirow[t]{2}{*}{ Variables } & \multirow[t]{2}{*}{ Patients $(n=169)$} & \multirow[t]{2}{*}{ Category or characteristics } & \multicolumn{3}{|c|}{ Univariate } & \multicolumn{3}{|c|}{ Multivariate } \\
\hline & & & HR & $95 \% \mathrm{CI}$ & $p$ value & HR & $95 \% \mathrm{CI}$ & $p$ value \\
\hline Gender & $19 / 150$ & (Female/male) & 1.12 & $0.550-2.687$ & 0.773 & & & \\
\hline Age & $59 / 110$ & $(<70 / \geq 70)$ & 1.93 & $1.205-3.061$ & 0.0066 & 2.024 & $1.255-3.236$ & 0.0042 \\
\hline TNM pStage & $101 / 68$ & $(1,2 / 3)$ & 2.725 & $1.714-4.349$ & $<0.0001$ & 2.51 & $1.555-4.065$ & 0.0002 \\
\hline Tumor size & $64 / 105$ & $(<3 / \geq 3)$ & 1.868 & $1.149-3.141$ & 0.0112 & 1.219 & $0.7364-2.083$ & 0.5582 \\
\hline Operation time & $111 / 58$ & $(<600 / \geq 600)$ & 1.481 & $0.9288-2.344$ & 0.0981 & & & \\
\hline Intraoperative blood loss & $87 / 82$ & $(<500 / \geq 500)$ & 1.308 & $0.825-2.098$ & 0.2541 & & & \\
\hline PNI & $98 / 71$ & $(\geq 49.2 /<49.2)$ & 2.612 & $1.600-4.405$ & $<0.0001$ & 2.248 & $1.362-3.832$ & 0.0013 \\
\hline $\mathrm{SCC}$ & $126 / 43$ & $(<1.5 / \geq 1.5)$ & 1.589 & $0.934-2.609$ & 0.0859 & & & \\
\hline
\end{tabular}

11.370; 95\% CI 4.040-47.500; $p<0.0001)$ as significant risk factors for shorter CSS. The TNM pStage (HR 3.488; 95\% CI 1.948-6.424; $p<0.0001$ ) and PNI (HR 3.849; $95 \%$ CI $1.987-8.205 ; p<0.0001)$ were confirmed as independent prognostic factors for CSS when multivariate logistic regression analysis was applied (Table 4).

Univariate analyses identified advanced TNM pStage (HR 3.772; 95\% CI 2.027-7.234; $p<0.0001$ ), large tumor size (HR 2.539; 95\% CI 1.315-5.298; $p=0.0050$ ), and low
PNI (HR 3.671; 95\% CI 1.864-7.897; $p=0.0001$ ) as significant risk factors for shorter OS. The TNM pStage (HR 2.615; 95\% CI 1.430-3.768; $p=0.0007$ ) and PNI (HR 2.275; 95\% CI 1.384-3.863; $p=0.001$ ) were confirmed as independent prognostic factors for OS in multivariate logistic regression analysis (Table 5).

Table 4 Univariate and multivariate analysis of cancer-specific survival in 110 non-elderly patients with esophageal cancer

\begin{tabular}{|c|c|c|c|c|c|c|c|c|}
\hline \multirow[t]{2}{*}{ Variables } & \multirow{2}{*}{$\begin{array}{l}\text { Patients } \\
(n=110)\end{array}$} & \multirow{2}{*}{$\begin{array}{l}\text { Category or } \\
\text { characteristics }\end{array}$} & \multicolumn{3}{|c|}{ Univariate } & \multicolumn{3}{|c|}{ Multivariate } \\
\hline & & & HR & $95 \%$ CI & $p$ value & HR & $95 \%$ CI & $p$ value \\
\hline Gender & $11 / 99$ & (Female/male) & 0.865 & $0.307-3.612$ & 0.8142 & & & \\
\hline TNM pStage & $64 / 46$ & $(1,2 / 3)$ & 4.646 & $2.281-10.027$ & $<0.0001$ & 3.488 & $1.948-6.424$ & $<0.0001$ \\
\hline Tumor size & $44 / 66$ & $(<3 / \geq 3)$ & 2.872 & $1.349-6.825$ & 0.0054 & 1.115 & $0.607-2.149$ & 0.7313 \\
\hline Operation time & $74 / 36$ & $(<600 / \geq 600)$ & 1.636 & $0.307-1.234$ & 0.1664 & & & \\
\hline $\begin{array}{l}\text { Intraoperative blood } \\
\text { loss }\end{array}$ & $55 / 55$ & $(<500 / \geq 500)$ & 1.176 & $0.591-2.371$ & 0.6437 & & & \\
\hline PNI & $61 / 49$ & $(\geq 49.2 /<49.2)$ & 11.37 & $4.040-47.500$ & $<0.0001$ & 3.849 & $1.987-8.205$ & $<0.0001$ \\
\hline SCC & $83 / 27$ & $(<1.5 / \geq 1.5)$ & 1.307 & $0.516-2.914$ & 0.5471 & & & \\
\hline
\end{tabular}


Table 5 Univariate and multivariate analysis of overall survival in 110 non-elderly patients with esophageal cancer

\begin{tabular}{|c|c|c|c|c|c|c|c|c|}
\hline \multirow[t]{2}{*}{ Variables } & \multirow[t]{2}{*}{ Patients $(n=110)$} & \multirow[t]{2}{*}{ Category or characteristics } & \multicolumn{3}{|c|}{ Univariate } & \multicolumn{3}{|c|}{ Multivariate } \\
\hline & & & HR & $95 \% \mathrm{CI}$ & $p$ value & HR & $95 \% \mathrm{CI}$ & $p$ value \\
\hline Gender & $11 / 99$ & (Female/male) & 1.08 & $0.390-4.478$ & 0.8968 & & & \\
\hline TNM Stage & $64 / 46$ & $(1,2 / 3)$ & 3.772 & $2.027-7.234$ & $<0.0001$ & 2.615 & $1.430-3.768$ & 0.0007 \\
\hline Tumor size & $44 / 66$ & $(<3 / \geq 3)$ & 2.539 & $1.315-5.298$ & 0.005 & 1.281 & $0.769-2.202$ & 0.3477 \\
\hline Operation time & $74 / 36$ & $(<600 / \geq 600)$ & 1.254 & $0.661-2.320$ & 0.4803 & & & \\
\hline Intraoperative blood loss & $55 / 55$ & $(<500 / \geq 500)$ & 1.524 & $0.822-2.911$ & 0.1821 & & & \\
\hline PNI & $61 / 49$ & $(\geq 49.2 /<49.2)$ & 3.671 & $1.864-7.897$ & 0.0001 & 2.275 & $1.384-3.863$ & 0.001 \\
\hline $\mathrm{SCC}$ & $83 / 27$ & $(<1.5 / \geq 1.5)$ & 1.515 & $0.697-3.028$ & 0.2787 & & & \\
\hline
\end{tabular}

\section{Prognostic factors for postoperative survival in elderly patients with ESCC}

In 59 elderly patients, univariate analyses demonstrated that the TNM pStage was the only significant risk factor for shorter CSS and OS (HR 3.701; 95\% CI 1.470-9.743; $p=0.0057$, HR $1.974 ; 95 \%$ CI 0.938-4.038; $p=0.0224$, respectively) (Table 6).

\section{PNI and postoperative survival in all patients with ESCC}

Kaplan-Meier analysis and the log-rank test demonstrated that patients with a low PNI had a significantly worse prognosis in terms of CSS and OS than those with a high PNI $(p<0.0001$, and $p=0.0003$, respectively). The 5-year CSS rates were 46.5 and $84.2 \%$, and the 5-year OS rates were 38.3 and $74.1 \%$ for patients with a low PNI and a high PNI, respectively (Fig. 2).

Table 6 Univariate and multivariate analysis of cancer-specific survival and overall survival in 59 elderly patients with esophageal cancer

\begin{tabular}{|c|c|c|c|c|c|c|c|c|}
\hline \multirow[t]{2}{*}{ Variables } & \multirow[t]{2}{*}{ Patients $(n=59)$} & \multirow[t]{2}{*}{ Category or characteristics } & \multicolumn{3}{|c|}{$\begin{array}{l}\text { Cancer-specific survival } \\
\text { Univariate }\end{array}$} & \multicolumn{3}{|c|}{$\begin{array}{l}\text { Overall survival } \\
\text { Univariate }\end{array}$} \\
\hline & & & HR & $95 \% \mathrm{CI}$ & $p$ value & HR & $95 \% \mathrm{CI}$ & $p$ value \\
\hline Gender & $8 / 51$ & (Female/male) & 1.85 & $0.526-11.717$ & 0.3755 & 1.577 & $0.615-5.349$ & 0.37 \\
\hline TNM pStage & $37 / 22$ & $(1,2 / 3)$ & 3.701 & $1.470-9.743$ & 0.0057 & 1.974 & $0.938-4.038$ & 0.0224 \\
\hline Tumor size & $20 / 39$ & $(<3 / \geq 3)$ & 0.973 & $0.390-2.623$ & 0.9542 & 1.073 & $0.526-2.313$ & 0.8507 \\
\hline Operation time & $37 / 22$ & $(<600 / \geq 600)$ & 1.909 & $0.768-4.815$ & 0.1614 & 1.731 & $0.859-3.491$ & 0.1235 \\
\hline Intraoperative blood loss & $32 / 27$ & $(<500 / \geq 500)$ & 0.689 & $0.265-1.714$ & 0.4235 & 1.023 & $0.506-2.088$ & 0.9493 \\
\hline PNI & $37 / 22$ & $(\geq 49.2 /<49.2)$ & 1.68 & $0.655-4.679$ & 0.2838 & 1.748 & $0.845-3.784$ & 0.1333 \\
\hline SCC & $43 / 16$ & $(<1.5 / \geq 1.5)$ & 1.218 & $0.387-3.287$ & 0.715 & 2.003 & $0.909-4.214$ & 0.0828 \\
\hline
\end{tabular}

Fig. 2 Kaplan-Meier curves of postoperative survival based on PNI in 169 patients with ESCC. a cancer-specific survival, b overall survival
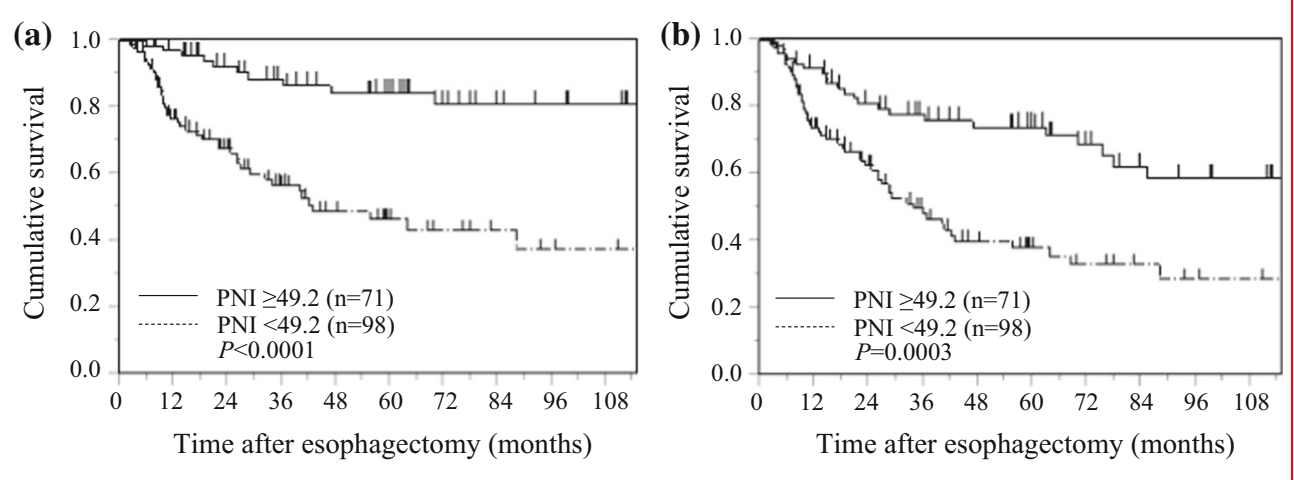


\section{PNI and postoperative survival in non-elderly patients with ESCC}

Kaplan-Meier analysis and the log-rank test demonstrated that patients with a low PNI had a significantly worse prognosis in terms of CSS and OS than those with a high PNI $(p<0.0001$, and $p=0.0003$, respectively). The 5 -year CSS rates were 44.1 , and $92.8 \%$ and the 5-year OS rates were 41.5 and $84.8 \%$ for patients with a low PNI and a high PNI, respectively (Fig. 3).

\section{PNI and postoperative survival in elderly patients with ESCC}

Kaplan-Meier analysis and the log-rank test showed no significant relationship between PNI and CSS $(p=0.1398)$ or OS ( $p=0.1907)$ in elderly patients with ESCC (Fig. 4).

\section{PNI and postoperative complications}

The postoperative complications in the high and low PNI groups are shown in Table 7. No significant differences in incidence rates of postoperative complication were observed between overall and age-stratified patients with a low versus high PNI. Similarly, there were no significant differences in postoperative hospital stay (data not shown).

\section{Discussion}

Treatment strategies for ESCC include surgery, radiation, chemotherapy, or a combination thereof. Although a complete surgical resection of the tumor offers a chance for cure, the rate of disease recurrence is very high in ESCC even after an aggressive surgery. Histopathology of surgical specimens is widely used to estimate the prognosis after surgery [12]; however, its predictive value is still limited. The precise evaluation of the risk of postoperative recurrence is important in planning a customized risk-adapted therapeutic strategy for individual patients. In particular, identifying prognostic factors prior to surgery is important to determine the optimal preoperative therapy, and to improve postoperative short- and long-term outcomes [16]. The original PNI consists of tests measuring albumin, transferrin, triceps skin fold, and skin sensitivity reaction to common antigens [17], and it was developed to assess the perioperative complications, including anastomotic leakage, delayed tissue repair, and length of hospital stay in patients undergoing gastrointestinal surgery $[11,18]$. The
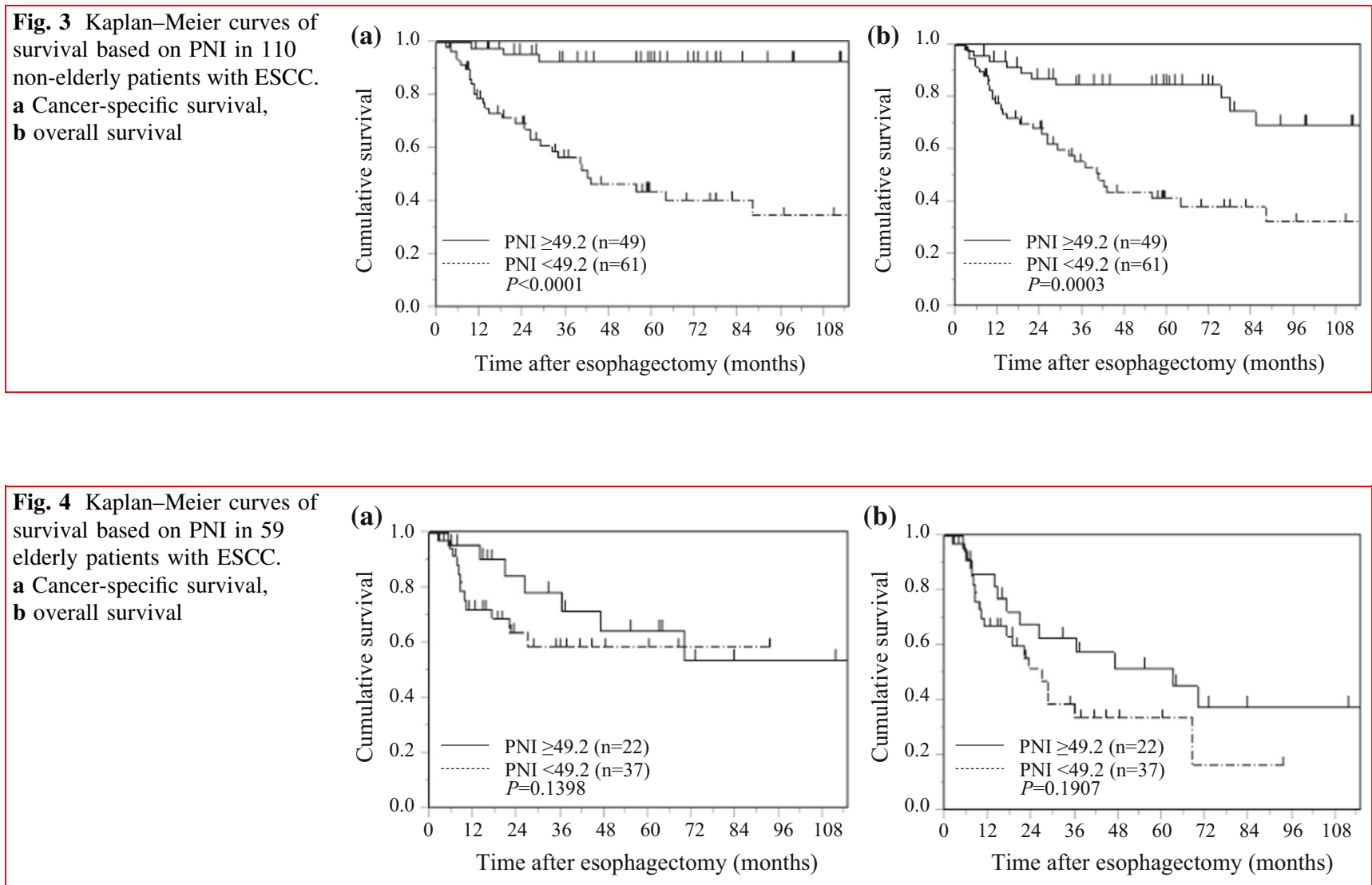


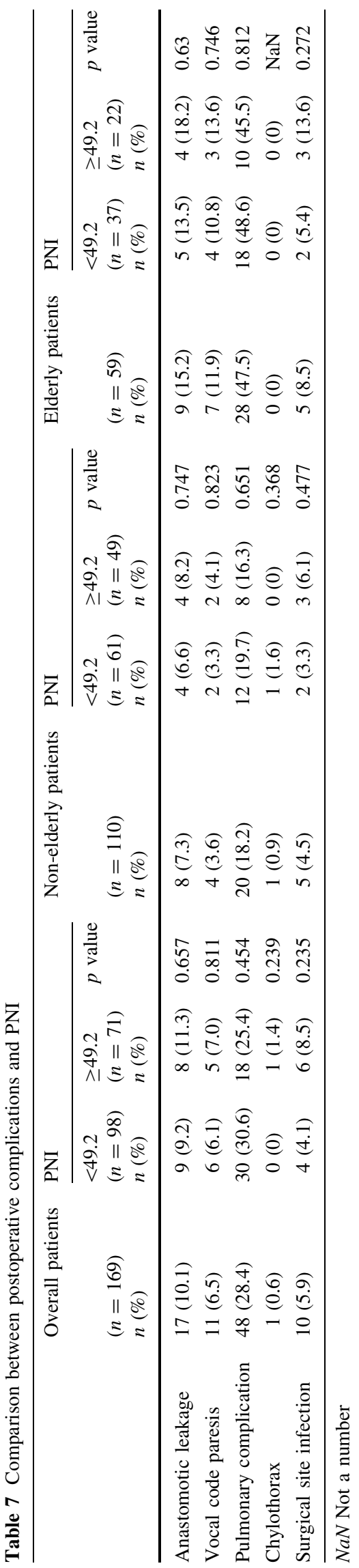

simplified PNI, on the other hand, consists of only two laboratory parameters, namely serum albumin concentration and total lymphocyte count in the peripheral blood [6], both of which are measured routinely in clinical practice. Recently, several studies have reported that albumin is produced by hepatocytes and is regulated by pro-inflammatory cytokines, including interleukin-1 (IL-1), IL-6, and tumor necrosis factor- $\alpha$ (TNF- $\alpha$ ) that adversely affect catabolic metabolism [19-21]. These cytokines produced by either the tumor itself or the host are crucial for carcinogenesis, cancer progression, and neo-angiogenesis. In addition, albumin has been shown to help stabilize cell growth and DNA replication, buffer a variety of biochemical changes and maintain sex hormone homeostasis to protect against tumorigenesis [10]. Albumin, therefore, is reflective of the inflammation and immune status of cancer, although it alone is not sufficient to predict the final outcome in cancer patients. Another element of the simplified PNI is the lymphocyte count. Lymphocytes are one of the fundamental components of cell-mediated immunity with inhibitory effects on the proliferation and invasion of tumor cells via cytokine-mediated cytotoxicity [11, 22]. Most patients with ESCC are malnourished, either due to poor dietary intake, protein loss from the primary lesion or due to catabolic metabolism [23]. Impaired nutritional and immunologic status accelerates tumor progression due to a decline in tumor immunity [16, 22]. The PNI is, therefore, a comprehensive indicator of the nutritional and immunologic status in cancer patients [24].

In this study, we evaluated the potential of simplified PNI as a prognostic marker of CSS and OS in ESCC patients who underwent radical thoracoscopic esophagectomy. Patients with a low PNI (less than 49.2) showed a significantly shorter CSS and decreased OS in comparison with patients who had a high PNI (49.2 or greater). This is consistent with our finding that PNI is inversely related to the stage of cancer, the value being lower in patients with a larger, deeper and more aggressive tumor at an advanced TNM pStage [25]. Recent studies have also demonstrated an association between the occurrences of postoperative complications with deteriorated CSS in patients with gastrointestinal cancer [26, 27]. Patients with a low PNI are, therefore, more likely to develop postoperative complications $[11,17,18]$, including a systemic inflammatory response, which can also contribute to the decreased CSS and OS. However, this correlation between PNI and CSS/ OS was found only in non-elderly patients and not in the elderly patients. This difference could be due to the fact that irrespective of being a cancer patient or not, hypoalbuminemia and leukocytopenia are often associated with aging, leading to malnutrition and immune suppression [28]. Previous studies have reported that cancer patients experiencing postoperative complications generally have a 
poorer prognosis [29-31]. Because patients with a low preoperative PNI value are at a high risk of postoperative complications, the preoperative PNI value may affect both postoperative short- and long-term outcomes. However, in this study, no significant differences were observed between the PNI value and the rate of postoperative complications. Therefore, further studies are thus needed to address this issue.

The optimal cutoff points for PNI in predicting postoperative survival in patients with malignant tumors is still controversial. Thus, one of the aims of this study was to evaluate the prognostic value of the PNI and propose an optimal cutoff, which can predict CSS and OS with better accuracy, in patients with ESCC. Based on our study of 169 patients who underwent curative esophagectomy, and using a ROC curve, we have arrived at 49.2 as the cutoff value for PNI. The cutoff value for PNI in ESCC patients seems to be much lower than those reported in another cancer, such as renal cell carcinoma, lung cancer, and gliomas [32-34]. The possible reason may be that most ESCC patients have some degree of swallowing difficulty due to obstruction or stricture of the esophagus, which results in the eating disorder, malnutrition, and subsequent poor nutritional status [35].

Several limitations should be considered when interpreting the results of this study. The retrospective nature and a single-institution study, the involvement of a comparatively small sample size, and short follow-up periods were the major limitations of this study. Minor limitations included insufficient evidence of the validity of the cutoff values for the PNI. Regarding the PNI, a fixed cutoff value has not yet been established, and various values have been used in previous reports. Another limitation was that some other parameters involved in systemic inflammation, such as TNF- $\alpha$ and interleukins were not studied, since, these inflammatory parameters are not routinely tested in clinical practice caused by its high cost and inconvenience.

Previous studies have shown that perioperative immunonutrition support improves both nutritional and immunologic status of patients undergoing elective surgeries, thereby reducing postoperative morbidity and mortality [9, 36]. However, it is still unknown whether such nutritional intervention can help improve the surgical outcomes in patients with a low PNI. A larger randomized multicenter prospective study is required to help determine this.

\section{Compliance with ethical standards}

Conflict of interest None of the authors have any conflicts of interest.

Ethical approval All procedures performed in the study involving human participants were in accordance with the ethical standards of the institutional and national research committee and with the 1964 Helsinki declaration and its later amendments or comparable ethical standards.

Informed consent Informed consent was obtained from all individual participants included in the study.

Open Access This article is distributed under the terms of the Creative Commons Attribution 4.0 International License (http://crea tivecommons.org/licenses/by/4.0/), which permits unrestricted use, distribution, and reproduction in any medium, provided you give appropriate credit to the original author(s) and the source, provide a link to the Creative Commons license, and indicate if changes were made.

\section{References}

1. Mainous MR, Deitch EA (1994) Nutrition and infection. Surg Clin N Am 74(3):659-676

2. Mellman I, Coukos G, Dranoff G (2011) Cancer immunotherapy comes of age. Nature 480(7378):480-489

3. Weimann A, Braga M, Harsanyi L et al (2006) ESPEN guidelines on enteral nutrition: surgery including organ transplantation. Clin Nutr 25:224-244

4. Oh SJ, Hyung WJ, Li C et al (2009) Effect of being overweight on postoperative morbidity and long-term surgical outcomes in proximal gastric carcinoma. J Gastroenterol Hepatol 24:475-479. https://doi.org/10.1111/j.1440-1746.2008.05704.x

5. Forrest LM, McMillan DC, McArdle CS et al (2004) Comparison of an inflammation-based prognostic score (GPS) with performance status (ECOG) in patients receiving platinum-based chemotherapy for inoperable non-small-cell lung cancer. $\mathrm{Br} \mathrm{J}$ Cancer 90:1704-1706

6. Onodera T, Goseki N, Kosaki G (1984) Prognostic nutritional index in gastrointestinal surgery of malnourished cancer patients. Nihon Geka Gakkai Zasshi 85:1001-1005 (in Japanese)

7. Nozoe T, Minomiya M, Maeda T et al (2010) Prognostic nutritional index: a tool to predict the biological aggressiveness of gastric carcinoma. Surg Today 40:440-443. https://doi.org/10. 1007/s00595-009-4065-y

8. Kanda M, Fujii T, Kodera Y et al (2011) Nutritional predictors of postoperative outcome in pancreatic cancer. $\mathrm{Br} \mathrm{J}$ Surg 98:268-274. https://doi.org/10.1002/bjs.7305

9. Smale BF, Mullen JL, Buzby GP et al (1981) The efficacy of nutritional assessment and support in cancer surgery. Cancer 47:2375-2381

10. Seaton K (2001) Albumin concentration controls cancer. J Natl Med Assoc 93:490-493

11. Gupta D, Lis CG (2010) Pretreatment serum albumin as a predictor of cancer survival: a systematic review of the epidemiological literature. Nutr J 9:69. https://doi.org/10.1186/1475-28919-69

12. Liu YP, Ma L, Wang SJ et al (2010) Prognostic value of lymph node metastases and lymph node ratio in esophageal squamous cell carcinoma. Eur J Surg Oncol 36:155-159. https://doi.org/10. 1016/j.ejso.2009.09.005

13. Dind D, Demartines N, Clavien PS (2004) Classification of surgical complications: a new proposal with evaluation in a cohort of 6336 patients and results of a survey. Ann Surg 240:205-213

14. Pohl H, Welch HG (2005) The role of over diagnosis and reclassification in the marked increase of esophageal adenocarcinoma incidence. J Natl Cancer Inst 97:142-146

15. Sobin LH, Gospodarowicz MK, Wittekind CH (2009) TNM classification of malignant tumours, 7th edn. Willey, Hoboken 
16. Lien YC, Hsieh CC, Wu YC et al (2004) Preoperative serum albumin level is a prognostic indicator for adenocarcinoma of the gastric cardia. J Gastointest Surg 8:1041-1048

17. Buzby GP, Mullen JL, Matthews DC et al (1980) Prognostic nutritional index in gastrointestinal surgery. Am J Surg 139:160-167

18. Schwegler I, von Holzen A, Gutzwiller JP et al (2010) Nutritional risk is a clinical predictor of postoperative mortality and morbidity in surgery for colorectal cancer. Br J Surg 97:92-97. https://doi.org/10.1002/bjs.6805

19. Mantovani A, Allavena P, Sica A et al (2008) Cancer-related inflammation. Nature 454:436-444. https://doi.org/10.1038/ nature 07205

20. Balkwill F (2009) Tumour necrosis factor and cancer. Nat Rev Cancer 9:361-371. https://doi.org/10.1038/nrc2628

21. Brenner D, Blaser H, Mak TW (2015) Regulation of tumour necrosis factor signalling: live or let die. Nat Rev Immunol 15:362-374. https://doi.org/10.1038/nri3834

22. Ray-Coquard I, Cropet C, Van Glabbeke M et al (2009) Lymphopenia as a prognostic factor for overall survival in advanced carcinomas, sarcomas, and lymphomas. Cancer Res 69:5383-5391. https://doi.org/10.1158/0008-5472.CAN-08-3845

23. Ryan AM, Reynolds JV, Healy L et al (2009) Enteral nutrition enriched with eicosapentaenoic acid (EPA) preserves lean body mass following esophageal cancer surgery: results of a doubleblinded randomized controlled trial. Ann Surg 249:355-363. https://doi.org/10.1097/SLA.0b013e31819a4789

24. Esper DH, Harb WA (2005) The cancer cachexia syndrome: a review of metabolic and clinical manifestations. Nutr Clin Pract 20:369-376

25. Coussens LM, Werb Z (2002) Inflammation and cancer. Nature 420:860-867

26. Yoo HM, Lee HH, Shim JH et al (2011) Negative impact of leakage on survival of patients undergoing curative resection for advanced gastric cancer. J Surg Oncol 104:734-740. https://doi. org/10.1002/jso.22045

27. Takeuchi D, Koide N, Suzuki A et al (2015) Postoperative complications in elderly patients with gastric cancer. J Surg Res 198:317-326. https://doi.org/10.1016/j.jss.2015.03.095
28. Ahmed T, Haboubi N (2010) Assessment and management of nutrition in older people and its importance to health. Clin Interv Aging 5:207-216

29. Aoyama T, Murakawa M, Katayama Y et al (2015) Impact of postoperative complications on sutvival and recurrence inpancreatic cancer. Anticancer Res 35:2401-2409

30. Kulu Y, Tarantio I, Warschkow R et al (2015) Anastomotic leakage is associated with impaired overall and disease-free survival after curative rectal cancer resction: a propensity score analysis. Ann Surg Oncol 22:2059-2067. https://doi.org/10.1245/ s10434-014-4187-3

31. Tokunaga M, Tanizawa Y, Bando E et al (2013) Poor survival rate in patients with postoperative intra-abdominal infectious complications following curative gastrectomy for gastric cancer. Ann Surg Oncol 20:1575-1583. https://doi.org/10.1245/s10434012-2720-9

32. Jeon HG, Choi DK, Sung HH et al (2016) Preoperative prognostic nutritional index is a significant predictor of survival in renal cell carcinoma patients undergoing nephrectomy. Ann Surg Oncol 23:321-327. https://doi.org/10.1245/s10434-015-4614-0

33. Shimizu K, Okita R, Saisho S et al (2015) Prognostic nutritional index before adjuvant chemotherapy predicts chemotherapy compliance and survival among patients with non-small-cell lung cancer. Ther Clin Risk Manag 8(11):1555-1561. https://doi.org/ 10.2147/TCRM.S92961

34. He ZQ, Ke C, Al-Nahari F et al (2017) Low preoperative prognostic nutritional index predicts poor survival in patients with newly diagnosed high-grade gliomas. $J$ Neurooncol 132(2):239-247. https://doi.org/10.1007/s11060-016-2361-0

35. Sun P, Zhang F, Chen C et al (2013) Comparison of the prognostic values of various nutritional parameters in patients with esophageal squamous cell carcinoma from Southern China. J Thorac Dis 5:484-491. https://doi.org/10.3978/j.issn.20721439.2013.08.38

36. Lai CC, You JF, Yeh CY et al (2011) Low preoperative serum albumin in colon cancer: a risk factor for poor outcome. Int $\mathrm{J}$ Colorectal Dis 26:473-481. https://doi.org/10.1007/s00384-010$1113-4$ 